\title{
Thoracolumbar Dorsal Ramus Nerve Block Using Continuous Multiorifice Infusion Catheters: A Novel Technique for Postoperative Analgesia After Scoliosis Surgery
}

\author{
JEFF L. XU, MD ${ }^{1}$, VICTOR TSENG, MD ${ }^{1}$, DAMON DELBELlO, MD², MATTHEW A. PRAVETZ, PHD ${ }^{3}$ \\ ${ }^{1}$ Division of Regional Anesthesia and Acute Pain Management, Department of Anesthesiology, Westchester Medical Center/New York Medical College, Valhalla, \\ New York, ${ }^{2}$ Department of Orthopedic Surgery, Westchester Medical Center/New York Medical College, Valhalla, New York, ${ }^{3}$ Department of Cell Biology and \\ Anatomy, New York Medical College, Valhalla, New York
}

\begin{abstract}
Background: This is a brief technical report about a novel regional anesthesia technique in which local anesthetic was deposited around the thoracolumbar dorsal rami nerves via 4 multiorifice pain catheters to obtain analgesia for posterior spinal fusion surgery on scoliosis patients. Scoliosis is the most common deformity of the spine. Currently, most surgeons prefer a dual rod, segmental spinal fixation system that allows multiple anchor points for attachment to the deformed spine. Scoliosis surgery is an extremely painful surgical procedure due to the large incision, surgical trauma to superficial and deep muscles of the back, and the insertion of pedicle screws and metal rods directly into the vertebral column. Postoperative pain management remains very challenging.

Methods: Three patients presented with scoliosis. Intraoperatively, 4 multiorifice catheters were placed lateral to the implanted pedicle screws. Two catheters were placed on each side, and a continuous infusion of $0.2 \%$ ropivacaine was initiated postoperatively to improve the patient's pain control. The catheters remained in place for 48 hours postoperatively and were removed by the surgical team. Gentle traction was applied similar to the way epidural catheters are removed.

Results: All 3 patients reported very low pain scores, low doses of opioid consumption, and satisfaction with their pain control throughout their hospitalization.

Conclusions: Our study results suggest that a thoracolumbar dorsal ramus nerve block using continuous multiorifice infusion catheters significantly improved postoperative comfort and pain and that its implementation into a multimodal analgesic regimen is relatively easy to achieve.
\end{abstract}

New Technology

Keywords: thoracolumbar dorsal ramus nerve block, multiorifice pain catheters, scoliosis surgery, posterior spinal fusion surgery, postoperative pain management

\section{INTRODUCTION}

Approximately 30000 spine fusions for scoliosis are performed in the United States every year, ${ }^{1,2}$ and most of these employ a dual rod, segmental spinal fixation. This technique allows multiple anchor points for correction of the deformed spine. These procedures require long incisions and extensive muscular dissection, with frequent multilevel bone osteotomies. As a result, patients commonly suffer from severe postoperative pain. Currently, postoperative pain management has been based primarily on a multimodal analgesic regimen composed of intravenous opioids, nonsteroidal anti-inflammatory drugs (NSAIDs), muscle relaxants, and epidural anesthesia. ${ }^{3}$ However, epidural anesthesia has significant limitations: including a tendency to cause hypotension, unnecessary blockage on the ventral ramus nerve that can cause significant motor and sensory blocks, limitations on postoperative neurological exam, and patchy and inadequate analgesic effect. We present a novel regional anesthetic technique in which local anesthetic is deposited around the thoracolumbar dorsal ramus nerve (TDRN) via 4 multiorifice pain catheters placed intraoperatively under direct visualization by the surgeon, which avoids many of the limitations encountered with epidurals.

\section{MATERIALS AND METHODS}

Three patients with adolescent idiopathic scoliosis (AIS) presented to the hospital for posterior spinal fusion and instrumentation (Table). All 3 
Table. Patient characteristics and total narcotic consumption.

\begin{tabular}{|c|c|c|c|}
\hline & Patient 1 & Patient 2 & Patient 3 \\
\hline Age, y & 16 & 11 & 13 \\
\hline Weight, kg & 54 & 60 & 41.8 \\
\hline Height, cm & 161 & 160 & 163 \\
\hline Cobb angle preoperative & $52^{\circ}$ & $42^{\circ}(\mathrm{T} 4-\mathrm{T} 11), 54^{\circ}(\mathrm{T} 11-\mathrm{L} 3)$ & $53^{\circ}(\mathrm{T} 5-\mathrm{T} 12), 54^{\circ}(\mathrm{T} 12-\mathrm{L} 4)$ \\
\hline Cobb angle postoperative & $9^{\circ}$ & $18^{\circ}(\mathrm{T} 4-\mathrm{T} 11), 15^{\circ}(\mathrm{T} 11-\mathrm{L} 3)$ & $10^{\circ}(\mathrm{T} 5-\mathrm{T} 12), 9^{\circ}(\mathrm{T} 12-\mathrm{L} 4)$ \\
\hline Instrumentation length, segments & T3-L4 & T4-L4 & T4-L4 \\
\hline Total narcotic MMEs, mg postoperative $48 \mathrm{~h}^{\mathrm{a}}$ & 20 & 0 & 34.5 \\
\hline
\end{tabular}

Abbreviation: MME, morphine milligram equivalent.

${ }^{\mathrm{a}}$ Hydromorphone patient-controlled analgesia converted at rate of $1 \mathrm{mg}$ hydromorphone, $5 \mathrm{mg}$ morphine.

patients did not have significant past medical histories other than AIS. Intraoperatively, each patient received general anesthesia with total intravenous anesthesia (propofol and remifentanil infusion) and with a titration of narcotics prior to extubation. For each patient, before wound closure, multiorifice "OnQ" catheters $(12.5 \mathrm{~cm}$, ON-Q Pain Relief System $($, I-Flow Corporation, Lake Forest, CA) were placed at the discretion of the attending orthopaedic surgeon. The catheters were placed laterally adjacent to the implanted pedicle screws under direct visualization (Figure 1a) to target the TDRNs at multiple vertebral levels. Our goal was to block sensory and motor innervation to the posterior muscles and skin with constant infusion

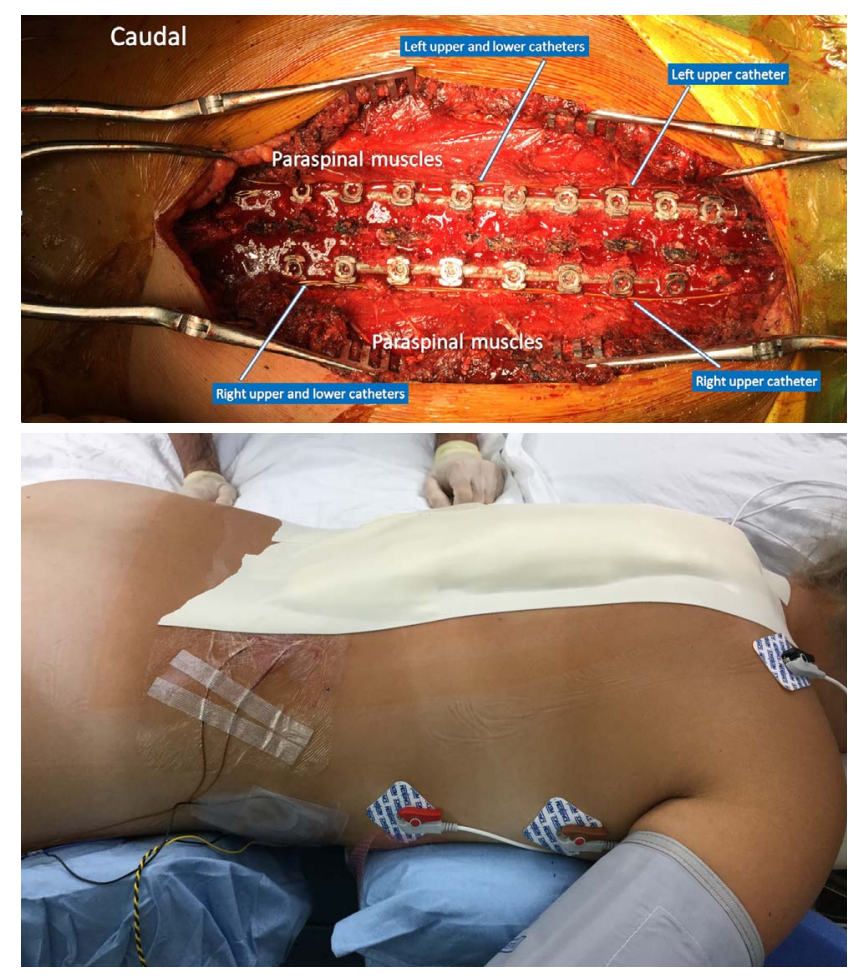

Figure 1. (a) Dorsal ramus nerve catheters prior to closure. (b) Dorsal ramus nerve catheters after closure (right side). of local anesthetic. We anticipated that 2 multiorifice catheters placed on each side (4 catheters total) would improve the spread of local anesthetic and the coverage of more vertebral levels, and thus provide efficient analgesia. The catheters were identified as left upper catheter (LUC), left lower catheter (LLC), right upper catheter (RUC), and right lower catheter (RLC). The 2 left-sided catheters (LUC and LLC) were connected by a "Stopcock" and sharing 1 infusion pump. The 2 right-sided catheters (RUC and RLC) were similarly connected by a Stopcock, sharing a separate infusion pump (Figure 1b). Immediately after extubation, patients received a bolus of $10 \mathrm{~mL}$ of $0.2 \%$ ropivacaine in each side, and a total of 2 infusions were started ( 1 for each side). The total infusion was initiated at $0.3 \mathrm{mg} / \mathrm{kg} / \mathrm{h}$ of $0.2 \%$ ropivacaine with titration up to $0.6 \mathrm{mg} / \mathrm{kg} / \mathrm{h}$. Titration adjustments were made by the intensive care unit (ICU) team based on the patients' reported level of pain. If the patient reported pain $\geq 7 / 10$, the infusion was increased by $1 \mathrm{~mL} / \mathrm{h}$ every hour bilaterally; if the patient reported pain $<3 / 10$, the infusion was reduced by $1 \mathrm{~mL} / \mathrm{h}$ every hour bilaterally. The TDRN block catheters remained in place for 48 hours postoperatively and were removed by the surgical team. Gentle traction was applied similar to the way epidural catheters are removed. In addition, patients received a multimodal analgesic regimen that included a hydromorphone patient-controlled analgesia (PCA) pump, 0.1 mg with 10 minute lockout (if necessary for breakthrough pain), NSAIDs, and Tylenol as part of a standard protocol.

\section{RESULTS}

No complications were noted during hospitalization or in the postoperative period. Patients reported their pain was well controlled. Patients were able to participate in physical therapy, eat, and 

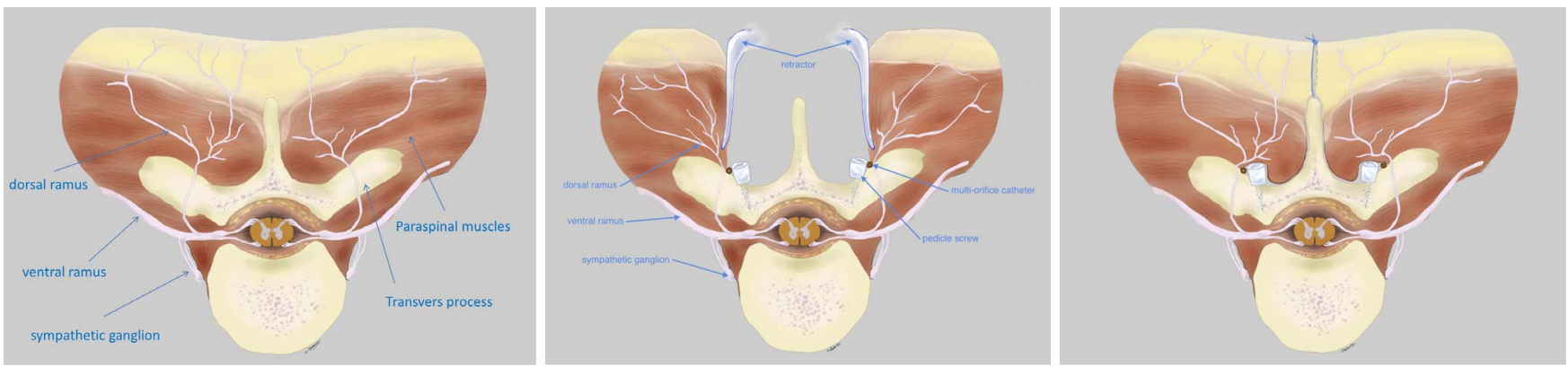

Figure 2. (a) Normal neuromuscular anatomy of the back. (b) Intraoperative placement of thoracolumbar dorsal ramus nerve catheters. (c) Postoperative relationship between thoracolumbar dorsal ramus nerve catheters and implanted pedicle screws.

sleep with comfort. There was no respiratory depression noted. The Foley catheters were removed on the same day of surgery. The patients' postoperative opiate use was recorded (Table), and it was noted that a markedly low dose of narcotics was required while the TDRN block catheters were in place.

\section{DISCUSSION}

It is well established ${ }^{4}$ that the dorsal rami of segmental spinal nerves supply the synovial joints of the vertebral column, the deep muscles of the back, and the overlying skin in a dermatomal pattern. After the segmental nerve exits the intervertebral foramen, it splits into dorsal and ventral rami. The dorsal rami then pass through a foramen bounded by the superior border of the transverse process, the anterior aspect of the superior zygapophysial (facet) joint, and the intertransverse ligament. ${ }^{5}$ Thus, it accesses the paraspinal muscles (multifidus, semispinalis, spinalis, longissimus, iliocostalis). Our technique targets the dorsal rami nerves as they exit the spinal canal, before they enter the paraspinal muscles. Repositioning the paraspinal muscles places the nerves in direct contact with the implanted catheters (Figures $2 \mathrm{a}-\mathrm{c}$ ).

The somatic pain resulting from tissue trauma as well as reflex muscle spasms makes the surgical correction of scoliosis an extremely painful procedure. Reviewing reports of pain management protocols subsequent to posterior spinal fusion have not resulted in an accepted "standard." who received the TDRN block catheters were pleased with their level of pain management and were able to mobilize quickly and comfortably. Subjectively, our ICU team reported that the patients with the TDRN block catheters had improved analgesia and comfort remarkably com- pared with patients who received a pain regimen that used an epidural catheter. Furthermore, patient 2 required no postoperative opiates while the catheters were in place and required a rescue PCA pump upon the TDRN block catheters' removal (a total of $6.6 \mathrm{mg}$ hydromorphone).

Patients who received TDRN block catheters required minimal opiates in the postoperative period and avoided affecting the blockage distribution of ventral ramus nerve and sympathetic chain. This allowed patients to participate in physical therapy as early as on postoperative day 0 . This bypassed the potential untoward side effects of epidural analgesia (hypotension, lower extremity weakness, respiratory depression, and urinary retention). In addition, the TDRN block catheters can be used in the upper thoracic spine without fear of respiratory depression and may be used in cases of wide laminectomy or large vertebrectomy. Given the results, the authors believe that a larger study comparing TDRN block catheters with the standard thoracic or lumbar epidurals should be performed in the setting of a multimodal analgesic regimen. We anticipate that the rate of complications will remain low ( 0 in this study) with good analgesic effect in this patient population, which has typically endured extraordinary discomfort in the postoperative period.

\section{ACKNOWLEDGMENTS}

The authors thank Victoria Y. Xu for permission to publish her digital drawing.

\section{REFERENCES}

1. Hresko MT. Idiopathic scoliosis in adolescents. $N$ Engl $J$ Med. 2013;368(9):834-841.

2. Noshchenko A, Hoffecker L, Lindley EM, et al. Predictors of spine deformity progression in adolescent 
idiopathic scoliosis: a systematic review with meta-analysis. World J Orthop. 2015;6(7):537-558.

3. Taenzer AH, Clark C. Efficacy of postoperative epidural analgesia in adolescent scoliosis surgery: a meta-analysis. Paediatr Anaesth. 2010;20(2):135-143.

4. Moore KL, Dalley AF, Agur AMR. 2014 Clinically Oriented Anatomy. 7th ed. Philadelphia, PA: Lippincott Williams and Wilkins.

5. Linqiu Z, Carson DS, Zhenhai S. The anatomy of dorsal ramus nerves and its implications in lower back pain. Neurosci Med. 2012;3(2):192-201.

6. Seki H, Ideno S, Ishihara T, Watanabe K, Matsumoto M, Morisaki H. Postoperative pain management in patients undergoing posterior spinal fusion for adolescent idiopathic scoliosis: a narrative review. Scoliosis Spinal Disord. 2018;13:17.

Disclosures and COI: The authors declare no conflict of interest; there are no financial or funding source(s) that support this work. Written informed consent was obtained from the patients for publi- cation of this brief technical report and any accompanying images.

Corresponding Author: Jeff L, Xu, MD, Division of Regional Anesthesia and Acute Pain Management, Department of Anesthesiology, Westchester Medical Center/New York Medical College, 100 Woods Road, Macy Building 2391, Valhalla, NY 10595. Phone: (914) 493-7799; Fax: (914) 4937927; Email: jeff.xu@wmchealth.org.

Published 30 April 2020

This manuscript is generously published free of charge by ISASS, the International Society for the Advancement of Spine Surgery. Copyright (c) 2020 ISASS. To see more or order reprints or permissions, see http://ijssurgery.com. 